\title{
Extreme thrombocytosis in a young cat
}

\author{
Emma Hooijberg • Ernst Leidinger • Georges Kirtz • \\ Mario Pichler
}

Received: 7 March 2010 / Accepted: 26 May 2010/Published online: 9 June 2010

(C) Springer-Verlag London Limited 2010

\begin{abstract}
A 7-month-old unvaccinated domestic shorthair cat was presented with a history of inappetence and hypersalivation. Clinical examination revealed fever, gingivitis and oral ulceration. An initial blood sample revealed a severe leukopenia and neutropenia and a feline panleukopenia virus (FPV) titre of 1:640; a second titre 10 days later was 1:2,560. A feline calicivirus (FCV) titre was 1:320 and the initial clinical signs and laboratory findings were attributed to a coinfection with FPV and FCV. The cat was treated with interferon omega. A blood sample taken 10 days later revealed a severe thrombocytosis of $3,448 \times 10^{9} / \mathrm{L}$ (reference range $200-500 \times 10^{9} / \mathrm{L}$ ) confirmed by blood smear examination. The platelet count on day 35 was $4,990 \times 10^{9} / \mathrm{L}$. No bone marrow examination was carried out. Two differential diagnoses for an extreme thrombocytosis are a reactive thrombocytosis or essential thrombocythemia (ET). ET is a hemic neoplasia that causes a severe and persistent thrombocytosis, and is a diagnosis of exclusion. Reactive thrombocytosis is generally mild to moderate and of shorter duration than described here, but an excessive response could have been caused by infection. The influence of additional factors such as an initial thrombocytopenia, age and interferon treatment are not clear but can be speculated on. Although the exact pathophysiology in this case remains unclear, such high, persistent thrombocyte numbers have not been reported in cats in the absence of a neoplastic process.
\end{abstract}

\footnotetext{
E. Hooijberg $(\triangle) \cdot$ E. Leidinger $\cdot$ G. Kirtz

Labor InVitro,

Rennweg 95,

Vienna 1030, Austria

e-mail: emma.hooijberg@invitro.at

M. Pichler

Tierklinik Meidling,

Vienna, Austria
}

Keywords Cat · Interleukin-6 · Panleukopenia · Platelets . Reactive thrombocytosis · Thrombopoietin

\section{Case presentation}

A 7-month-old, male neutered domestic shorthair cat presented to his veterinarian with a complaint of inappetence and a swaying gait. The cat was allowed to go outside and was unvaccinated. The cat shared a household with a female littermate that had received only the first part of the primary vaccination 3 months previously.

Upon examination, the cat was found to be pyrexic (temperature $41^{\circ} \mathrm{C}$ ), and an examination of the oral cavity revealed an ulcerative gingivostomatitis. The gait abnormality was identified by the veterinarian as a hindlimb ataxia.

A blood sample was collected and sent to the InVitro Diagnostic Laboratory. Hematology was performed (CELLDYN. 3500 with Veterinary Package Software, Abbott Laboratories, Abbott Park, IL, USA) and revealed severe leukopenia (WBC $1.0 \times 10^{9} / \mathrm{L}$, reference interval $6.0-15.0 \times$ $\left.10^{9} / \mathrm{L}\right)$ with severe neutropenia $\left(0.2 \times 10^{9} / \mathrm{L}\right.$, reference interval $\left.3.6-10.5 \times 10^{9} / \mathrm{L}\right)$ and mild lymphopenia $\left(0.7 \times 10^{9} / \mathrm{L}\right.$, reference interval $\left.1.0-3.2 \times 10^{9} / \mathrm{L}\right)$. The platelet count was $314 \times 10^{9} / \mathrm{L}$ (reference interval $200-500 \times 10^{9} / \mathrm{L}$. Clinical chemistry analysis (Dade Behring Dimension RxL, Dade Behring, Marburg Germany) showed hyperproteinemia (85 g/L, reference interval 60-75 g/L) and hyperglobulinemia $(54 \mathrm{~g} / \mathrm{L}$, reference interval $35-42 \mathrm{~g} / \mathrm{L})$.

An immunofluorescent antibody test (IFAT) (MegaScreen FLUO CPV, Megacor Diagnostik, Hörbranz, Austria) for feline parvovirus was performed and was positive at a titre of 1:640 (borderline titre 1:80). A tentative diagnosis of feline panleukopenia was made and 
treatment was started with interferon omega therapy (Virbagen ${ }^{\circledR}$ Omega, Virbac, Vienna, Austria).

The female littermate presented 5 days later with fever and oral ulceration, and had a feline parvovirus titre of 1:1280. She was also given interferon omega therapy.

Both cats improved clinically; although the hindlimb ataxia in the male cat improved, it did not resolve. A blood sample for routine hematology and biochemistry was taken from the male patient for monitoring purposes 10 days after the initial sample. A second feline parvovirus IFAT was positive at a titre of 1:2560, the fourfold increase in titre confirming the diagnosis of feline panleukopenia. A feline calicivirus (FCV) titre (MegaScreen FLUO FCV, Megacor Diagnostik, Hörbranz, Austria) was also performed at this time, and the positive result (titre 1:320, borderline titre 1:40) together with the oral ulceration seen in both cats supported a coinfection with FCV.

A third sample was analysed 35 days after initial sampling. The results of the samples from day 10 and day 35 are presented in Table 1.

Tests for feline leukemia virus and feline corona virus performed on day 0 and for Toxoplasma gondii and feline herpes virus performed on day 10 were negative.

A manual platelet estimation performed on a blood smear, using a method previously described by Tasker et al. (1999), supported the automated platelet count found on day 10 .

The blood smear examined on day 10 is shown in Figs. 1 and 2.
Platelets were distributed evenly throughout the smear and platelet clumping was minimal. Platelet size varied, with small numbers of platelets larger than erythrocytes present (megaplatelets). Many platelets showed partial activation according to a description by Harvey (2001). No abnormal forms or megakaryocytes were seen.

The automated platelet count measured by the Cell Dyn on day 35 was $2,390 \times 10^{9} / \mathrm{L}$. A manual platelet count using a Neubauer hemocytometer gave a result of $4,990 \times 10^{9} / \mathrm{L}$. A blood smear was also examined and findings were similar to that of day 10. As the original measured red cell count on day 35 was raised and the mean cell volume (MCV) decreased, it was assumed that the Cell Dyn had erroneously counted a large number of platelets as red blood cells, as has been previously reported (Green 1999). Red blood cell parameters were accordingly corrected (corrected values are displayed in Table 1).

A bone marrow examination was suggested, but permission was not granted by the owner.

Blood samples from the female cat revealed no abnormalities apart from a mild thrombocytosis $\left(700 \times 10^{9} / \mathrm{L}\right.$, Cell Dyn 3500 ), confirmed by a smear examination.

The male cat developed no further clinical signs. A fourth and final blood sample taken 4 months after initial presentation revealed a mild persistent thrombocytosis $\left(714 \times 10^{9} / \mathrm{L}\right.$, Cell Dyn 3500 , confirmed by a smear examination).
Table 1 Laboratory data day 10 and day 35 after initial sampling

\begin{tabular}{llll}
\hline Analyte & Day 10 & Day 35 & Reference interval \\
\hline Hematocrit & 0.29 & 0.38 & $0.28-0.47$ \\
Red blood cell count $\left(\times 10^{12} / \mathrm{L}\right)$ & 6.8 & 8.5 & $5.5-10.0$ \\
Hemoglobin $(\mathrm{g} / \mathrm{L})$ & 100 & 144 & $80-170$ \\
Mean cell volume $(\mathrm{fL})$ & 42 & 44 & $40-55$ \\
Mean cell hemoglobin concentration $(\mathrm{g} / \mathrm{L})$ & $\mathbf{3 5 0}$ & $\mathbf{3 8 0}$ & $310-340$ \\
WBC $\left(\times 10^{9} / \mathrm{L}\right)$ & 10.8 & $\mathbf{1 7 . 6}$ & $6.0-15.0$ \\
Band neutrophils $\left(\times 10^{9} / \mathrm{L}\right)$ & 0.0 & 0.2 & $0.0-0.6$ \\
Segmented neutrophils $\left(\times 10^{9} / \mathrm{L}\right)$ & 7.6 & 9.5 & $3.6-10.5$ \\
Lymphocytes $\left(\times 10^{9} / \mathrm{L}\right)$ & 2,2 & $\mathbf{3 . 9}$ & $1.0-3.2$ \\
Monocytes $\left(\times 10^{9} / \mathrm{L}\right)$ & 0.8 & $\mathbf{1 . 4}$ & $0.0-0.6$ \\
Eosinophils $\left(\times 10^{9} / \mathrm{L}\right)$ & 0.1 & $\mathbf{2 . 5}$ & $0.0-0.6$ \\
Basophils $\left(\times 10^{9} / \mathrm{L}\right)$ & 0.1 & 0.2 & $0.0-0.3$ \\
Platelets $\left(\times 10^{9} / \mathrm{L}\right)$ & $\mathbf{3 , 4 4 8}$ & $\mathbf{4 , 9 9 0}$ & $200-450$ \\
Total protein $(\mathrm{g} / \mathrm{L})$ & Not performed & $\mathbf{7 9}$ & $60-75$ \\
Albumin $(\mathrm{g} / \mathrm{L})$ & Not performed & 30 & $26-36$ \\
Globulin $(\mathrm{g} / \mathrm{L})$ & Not performed & $\mathbf{4 9}$ & $35-42$ \\
Iron $(\mu \mathrm{mol} / \mathrm{L})$ & Not performed & 1.8 & $1.8-3.2$ \\
FPV $(\mathrm{IFAT})$ & $1: 2560$ & Not performed & Borderline titre $1: 80$ \\
FCV $(\mathrm{IFAT})$ & $1: 320$ & Not performed & Borderline titre $1: 40$ \\
\hline
\end{tabular}




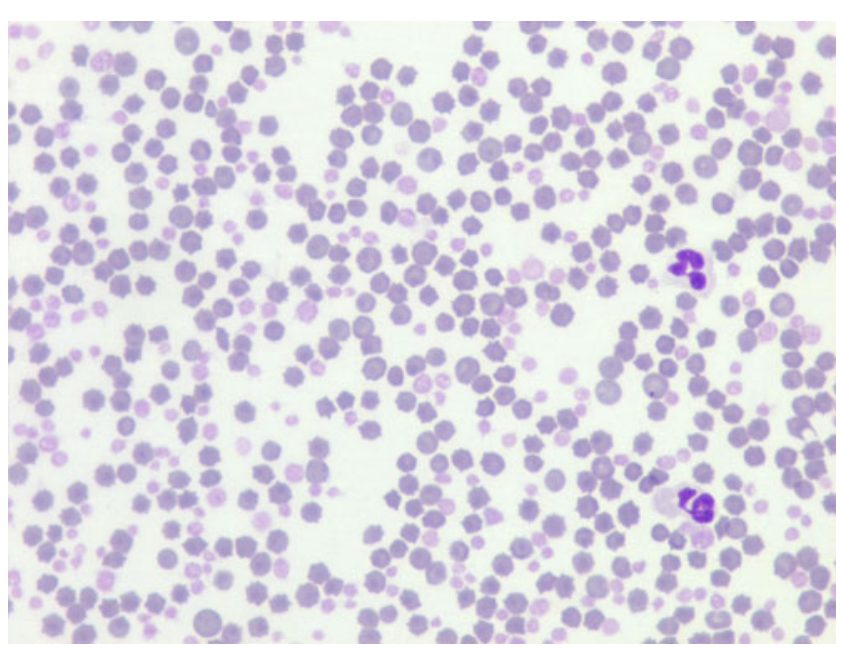

Fig. 1 Blood smear from the male cat showing a marked thrombocytosis. Modified Wright-Giemsa, $\times 40$ objective

\section{Discussion}

The number of platelets in circulation in a healthy animal depends on negative and positive feedback mechanisms and on the rate of their production, consumption and destruction. Platelet production is regulated mainly by thrombopoietin (Tpo), which causes the lineage-specific differentiation of hematopoietic stem cells into committed megakaryocytic progenitor cells, and the proliferation and further differentiation of megakaryoblasts to megakaryocytes (Dame and Sutor 2005). Tpo is produced primarily by hepatocytes but also by renal tubular cells and bone marrow stromal cells (Kaushansky 2005).

A negative feedback loop exists between the levels of Tpo and the number of platelets: circulating Tpo binds to and is taken up by platelets and destroyed. A greater mass of platelets will therefore result in lower levels of Tpo

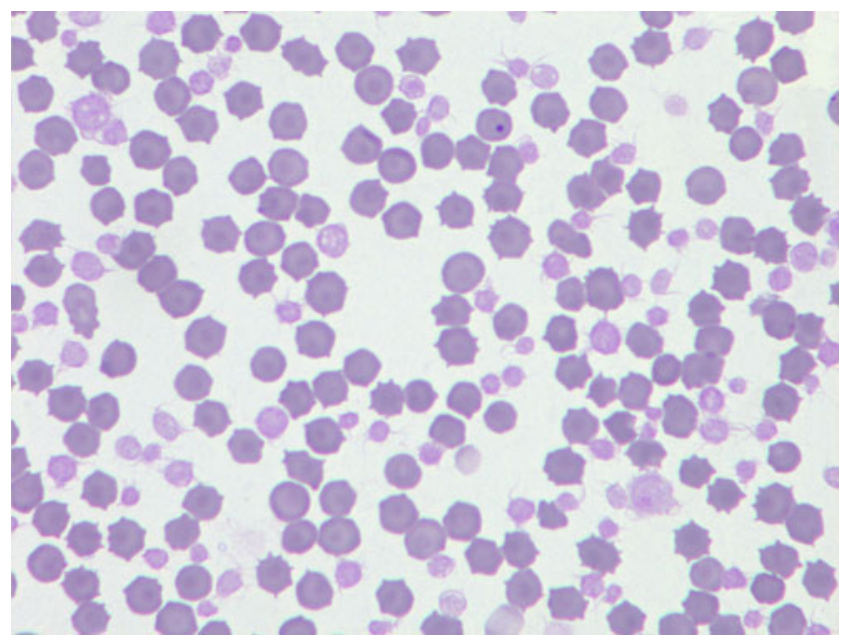

Fig. 2 Higher magnification of the blood smear. Some megaplatelets are present. Modified Wright-Giemsa, $\times 100$ objective which in turn will result in decreased platelet production. In thrombocytopenic states, lower numbers of platelets mean less Tpo binding, uptake and destruction, leading to higher Tpo levels and thus increased thrombopoiesis (Kaushansky 2005). This process is illustrated in Fig. 3.

Interleukin-6 (IL-6), a cytokine released from monocytes and macrophages during inflammation, has been shown to induce the production of Tpo in hepatocytes (Kaser et al. 2001).

The two differential diagnoses that could be considered for an extreme thrombocytosis are essential thrombocythemia (ET) and a reactive thrombocytosis.

ET is a rare chronic myeloproliferative disease characterized by the clonal proliferation of the megakaryocytic system resulting in a marked and persistent thrombocytosis in the blood. ET has been reported in four dogs, one cat and suspected in a horse (Bass and Schultze 1998; Dunn et al. 1999; Hammer et al. 1990; Hopper et al. 1989; Sellon et al. 1997). Platelet counts in the reports concerning dogs and the cat ranged from $925 \times 10^{9} / \mathrm{L}$ to $4,950 \times 10^{9} / \mathrm{L}$ and were accompanied by an anemia, either regenerative or nonregenerative. ET is a diagnosis of exclusion; in humans, a scheme of diagnostic criteria released by the Polycythemia Study Group is used (Mandell 2000). These criteria include a platelet count of greater than $600 \times 10^{9} / \mathrm{L}$, a hematocrit less than 0.4 (i.e. anemia), stainable bone marrow iron/ normal serum ferritin, no or minimal bone marrow fibrosis, no evidence for a myelodysplastic syndrome or cause for reactive thrombocytosis.

A bone marrow sample could unfortunately not be obtained, and feline serum ferritin measurements are not available in Europe so the iron status of the cat could not be fully ascertained. Serum iron levels measured on day 35 were low normal. Iron deficiency has been associated with reactive thrombocytosis in humans; the pathogenesis is unclear (Dan 2005).

As a clear temporal relationship between the viral infections and the thrombocytosis existed, a diagnosis of reactive thrombocytosis was made. The age of the animal, absence of abnormal platelets on the blood smear and continuing survival of the cat without the development of any additional clinical signs further support the unlikelihood of ET in this case.

Various inflammatory conditions can result in a reactive thrombocytosis. A number of cytokines are released from monocytes and macrophages during inflammation, including IL-6. IL-6 has been shown to induce the production of Tpo by hepatocytes (Kaser et al. 2001). This leads to an increase in the thrombocyte count due to the effect of increased levels of Tpo on platelet production. In humans, IL-6 levels have been shown to be raised in patients with reactive thrombocytosis (Dan 2005; Schafer 2004). 
Fig. 3 The relationship between thrombopoietin and platelet production. High Tpo levels lead to an increase in platelet mass. A greater platelet mass results in more Tpo being removed from circulation by the platelets. The resulting decrease in Tpo levels leads to a decrease in platelet mass and consequently an increase in Tpo. In health, Tpo is produced at a constant rate by hepatocytes; cytokines especially IL-6 stimulate increased production of Tpo

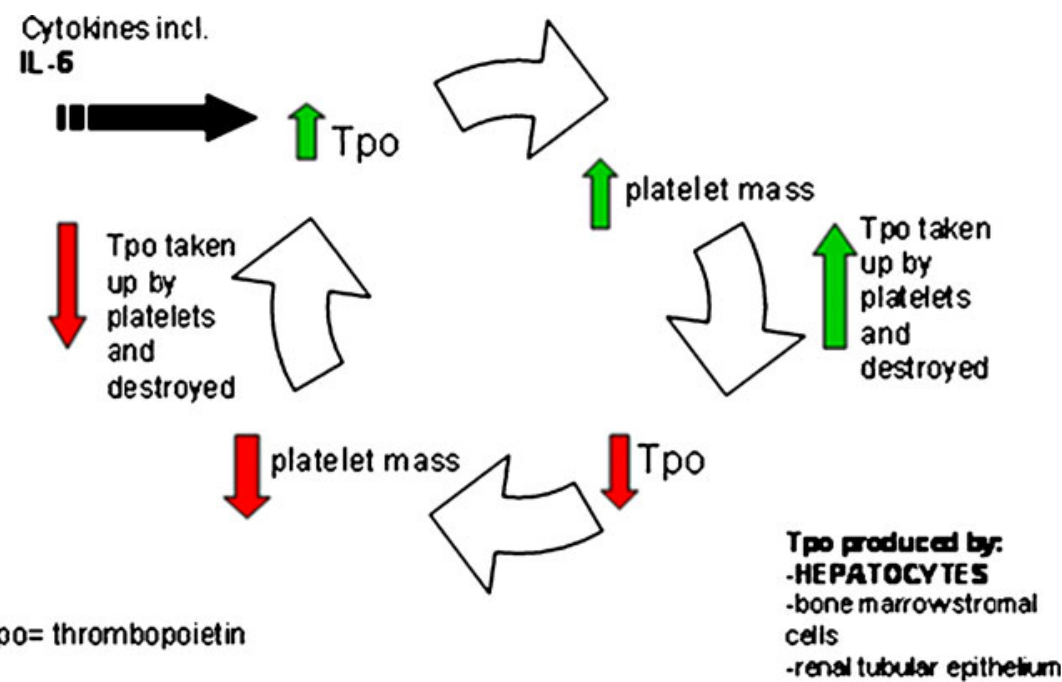

Reactive thrombocytosis has been described in cats, dogs, horses and other animal species, and two retrospective studies have been published concerning reactive thrombocytosis in cats (Hammer 1991; Rizzo et al. 2007). In the earlier study by Hammer (1991), in which results from 17 cats were examined, the most common causes overall were found to be neoplastic, gastrointestinal, endocrine, urinary and hematological disorders. In the more recent study by Rizzo et al. (2007) of thrombocytosis in 51 cats, it was found that reactive thrombocytosis occurred most commonly secondary to gastrointestinal, endocrine, cardiovascular, urinary, respiratory, hematological and musculoskeletal diseases. None of the diagnoses specifically mentioned feline parvovirus; however, there was a case of calicivirus in the latter study. Reactive thrombocytosis has also been reported in neoplasia and due to corticosteroid and antineoplastic drug use (Jain 1993).

In the study involving 17 cats, the mean platelet count was $767 \times 10^{9} / \mathrm{L}$ and two cats had platelet counts above $1,000 \times 10^{9} / \mathrm{L}$ (Hammer 1991). The maximum platelet count found in these cats is not mentioned. Viral enteritis is recorded as a cause for a platelet count of greater than $1,000 \times 10^{9} / \mathrm{L}$ in this study; however, whether this was a dog or cat is not clear.

In the second study concerning 51 cats, the median platelet count was $801 \times 10^{9} / \mathrm{L}$ with a maximum count of $1,895 \times 10^{9} / \mathrm{L}$ (Rizzo et al. 2007). In this more recent study, the platelet count is reported only once for each patient and no information regarding duration of the abnormality was gathered. No reports of platelet counts in a cat as high as
Fig. 4 Possible mechanisms contributing to the increase in platelet numbers in this cat. A transient thrombocytopenia resulting from feline panleukopenia virus (FPV) infection and interferon omega treatment leads to an increase in Tpo due to its decreased removal from circulation by the decreased platelet mass. IL- 6 production caused by inflammation relating to FPV and FCV further increases Tpo levels which in turn leads to a large increase in platelet mass. A possible age-related sensitivity to Tpo may contribute to the extreme increase in platelets

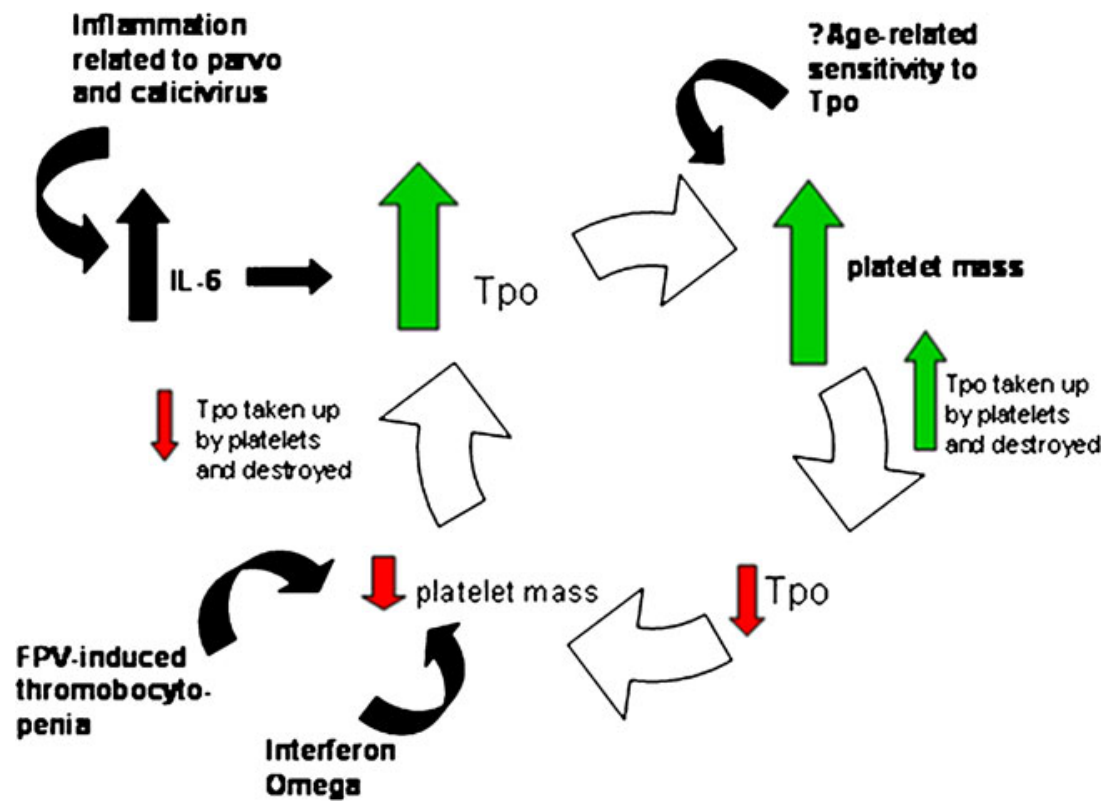


those recorded in the case here could be found. In humans, the majority of platelet counts in patients with reactive thrombocytosis are below $1,000 \times 10^{9} / \mathrm{L}$, but counts higher than this have been reported (Buss et al. 1994).

In the earlier study, the mean age of cats with thrombocytosis was 8.3 years old and there was no statistical difference in platelet numbers between age groups. In the more recent study, however, $25.4 \%$ of the cases were younger than 1 year old, but an association between young age and thrombocytosis was not confirmed. In humans, reactive thrombocytosis shows an agedependant pattern with the highest incidence occurring in children under 2 years of age (Dame and Sutor 2005). Suggested causes include increased expression of Tpo in the bone marrow and increased sensitivity of progenitor cells to Tpo.

The reasons for the extremely high platelet counts found in this cat can only be speculated on and several factors may be involved. It is highly likely that an unusually high level of Tpo was the primary stimulus for increased platelet production. Figure 4 illustrates how these factors may have caused an increase in Tpo and thus platelet numbers.

As well as the typical panleukopenia, which occurs 46 days post infection, feline parvovirus infection can also cause a thrombocytopenia (Jain 1993). This cat was treated with interferon omega, which has been described as causing a mild and transient thrombocytopenia in clinical trials in cats (Information from European Medicines Agency Virbagen Omega Scientific Discussion 2009). The life span of platelets is 5-10 days and thus a decrease in platelet count to below normal levels would take longer to occur than neutropenia after a bone marrow insult (Stockham and Scott 2008). It is possible that a transient thrombocytopenia was present after day 0 but before day 10 as a result of the parvovirus and/or interferon omega, but as no blood sample was taken during this time, this cannot be proven. Theoretically decreased platelet numbers would have resulted in an increase in Tpo levels as explained in Figs. 3 and 4 and a rebound thrombocytosis. Platelet numbers can increase rapidly-in a study concerning rats with induced thrombocytopenia, maximum rebound thrombocytosis (platelet count $1,720 \times$ $10^{9} / \mathrm{L}$ ) occurred 4-5 days after the lowest platelet count $\left(101 \times 10^{9} / \mathrm{L}\right)$ was recorded (Kimura et al. 1985). Data from a splenectomized dog with a normal platelet count $\left(377 \times 10^{9} /\right.$ L) pre-splenectomy and a platelet count of $1,197 \times 10^{9} / \mathrm{L}$ 1 day and $1,395 \times 10^{9} / \mathrm{L} 5$ days post-splenectomy indicate that platelet numbers can increase greatly in a very short time and that an increase from a theoretical thrombocytopenia in the first few days following day 0 to a platelet count of $3,448 \times 10^{9} / \mathrm{L}$ on day 10 is plausible (Jain 1993). However, it is probably unlikely that a thrombocytopenia alone would result in such high platelet numbers.
An inflammatory process was present in this case, which would have lead to increased production and release of IL-6 which in turn leads to increased Tpo production. Whether particularly high IL-6 levels are related to feline parvovirus or calicivirus infection is not known. The nonstructural protein (NS1) of human parvovirus B19 has been shown to directly cause increased expression and secretion of IL-6 (Hsu et al. 2006; Mitchell 2002). Unfortunately, no assays for feline Tpo or IL-6 are commercially available, and although measurement of these substances may have been interesting, lack of reference data would make interpretation difficult.

Thus, high levels of thrombopoietin due to increased production related to raised IL- 6 and decreased consumption as a result of a transient thrombocytopenia, as well as an age-related sensitivity to Tpo, may have caused the unusually extreme reactive thrombocytosis seen here. Interestingly, the blood samples of the littermate of this patient that displayed similar clinical signs and received the same treatment displayed only a mild thrombocytosis $\left(700 \times 10^{9} / \mathrm{L}\right.$, Cell Dyn 3500 , confirmed on blood smear $)$ on hematological examination. Further reports of extreme thrombocytosis in young animals and subsequent to viral infections may be useful in explaining the findings documented here.

\section{References}

Bass MC, Schultze AE (1998) Essential thrombocythemia in a dog: case report and literature review. J Am Anim Hosp Assoc 34:197-203

Buss DH, Cashell AW, O'Connor ML et al (1994) Occurrence, etiology, and clinical significance of extreme thrombocytosis: a study of 280 cases. Am J Med 96:247-253

Dame C, Sutor AH (2005) Primary and secondary thrombocytosis in childhood. Br J Haematol 129:165-177

Dan K (2005) Thrombocytosis in iron deficiency anaemia. Intern Med 44:1025-1026

Dunn JK, Heath MF, Jefferies A et al (1999) Diagnostic and hematologic features of probable essential thrombocythemia in two dogs. Vet Clin Pathol 28:131-138

Green RA (1999) Spurious platelet effects on erythrocyte indices using the Cell-Dyn 3500 automated hematology system. Vet Clin Pathol 28:47-49

Hammer AS (1991) Thrombocytosis in dogs and cats: a retrospective study. Comp Haematol Int 1:181-186

Hammer AS, Couto CG, Getzy D et al (1990) Essential thrombocythemia in a cat. $\mathrm{J}$ Vet Intern Med 4:87-91

Harvey J (2001) Atlas of veterinary hematology. WB Saunders, Philadelphia, p 77

Hopper PE, Mandell CP, Turrel JM et al (1989) Probable essential thrombocythemia in a dog. $\mathrm{J}$ Vet Intern Med 3:79-85

Hsu T-C, Tzang B-C, Huang C-N (2006) Increased expression and secretion of interleukin- 6 in human parvovirus B19 nonstructural protein (NS1) transfected COS-7 epithelial cells. Clin Exp Immunol 44:152-157 
Information from European Medicines Agency Virbagen Omega Scientific Discussion. http://www.emea.europa.eu/vetdocs/PDFs/ EPAR/virbagenomega/108501en6.pdf. Accessed 4 August 2009

Jain N (1993) Essentials of veterinary hematology. Lea and Febiger, Media, pp 115-125

Kaser A, Brandacher G, Steurer W et al (2001) Interleukin-6 stimulates thrombopoiesis through thrombopoietin: role in inflammatory thrombocytosis. Blood 98:2720-2725

Kaushansky K (2005) The molecular mechanisms that control thrombopoiesis. J Clin Invest 115:3339-3347

Kimura H, Segal GM, Lee MY (1985) Megakaryocytopoiesis in the rat: response to thrombocytopenia induced by exchange transfusion [abstract]. Exp Hematol 13:1048-1054

Mandell CP (2000) Essential thrombocythemia and reactive thromocytosis. In: Feldman BF, Zinkl JG, Jain NC (eds) Schalm's veterinary hematology, 5th edn. Blackwell Publishing, Ames, pp 501-508
Mitchell LA (2002) Parvovirus B19 Nonstructural (NS1) Protein as transactivator of interleukin-6 synthesis: common pathway in inflammatory sequelae of human parvovirus infections? J Med Virol 67:267-274

Rizzo F, Tappin SW, Tasker S (2007) Thrombocytosis in cats: a retrospective study of 51 cases (2000-2005). J Feline Med Surg 9:319-325

Schafer AI (2004) Thrombocytosis. N Engl J Med 350:12111219

Sellon DC, Levine JF, Palmer K et al (1997) Thrombocytosis in 24 horses (1989-1994). J Vet Intern Med 11:24-29

Stockham SL, Scott MA (2008) Fundamentals of veterinary clinical pathology, 2nd edn. Blackwell Publishing, Aimes, p 227

Tasker S, Cripps PJ, Mackin AJ (1999) Estimation of platelet counts on feline blood smears. Vet Clin Pathol 28:42-45 